\title{
Understanding the tourist mobility using GPS: where is the next place?
}

\begin{abstract}
Understanding the mobility of tourists plays a fundamental role in the administration and design of tourist destinations, planning of on-site movement and marketing of attractions. In this paper, we focus on how to accurately predict the tourist's next location within a given attraction. A heuristic method based on data mining is proposed, which considers the trajectory of a focal tourist and the movements of past visitors. To evaluate the performance of the proposed method, a case study was conducted at the Summer Palace in Beijing, China. We collected movement information from tourists using GPS tracking technology, and the results of an independent samples t-test indicate that the proposed method indeed performs significantly better than existing methods. We further explore the potential applications of the proposed method. Our results significantly contribute to enhancing the level of personalized location-based service, tourist attraction administration, and real-time crowd control.
\end{abstract}

Keyword: Tourist mobility; prediction; GPS technology; data mining

\section{Introduction}

Tourism is generally regarded as separate from daily life; therefore the study of tourism has often been seen as peripheral to the social sciences. However, the mobility paradigm arguably allows us to place tourism at the core of social and cultural life rather than at the margins (Coles \& Hall, 2006). The formation, development and disappearance of mobility in physical space is an important way for humans to experience the world, the sense of space production and the progress of place formation, including the social forces associated with almost all complex and related information, such as the mobility of people, objects and information (Cresswell, 2006). The focus on mobility allows us to show how the discourses and practices of freedom implied by driving underline the contemporary tourism experience in some contexts (Hannam, et al., 2014). Proponents of the mobility paradigm argue that the concept of mobility is concerned with mapping and understanding not only the large-scale movements of people, objects, capital and information across the world, but also the more local simultaneous processes of daily transportation, passage through public space and the movement of material things in everyday life (Hannam, et al., 2006).

Understanding tourist mobility plays a fundamental role in the administration and design of destinations, planning of on-site movement and marketing of attractions (Xia, et al., 2011). In the past few years, academics and practitioners have increasingly studied the movement 
patterns of tourists (De Cantis, et al., 2016; Xia, et al., 2009; Xia, et al., 2011), and how to guide practice based on this movement pattern, for example, by using market segmentation (Xia, et al., 2010) and behaviour analysis (Huang \& Wu, 2012; McKercher, et al., 2015).

With the recent development of mobile Internet technology and geographic information technology, it is possible to track and record the space-time path of individual tourists accurately (Shoval \& Isaacson, 2007). However, tracking and recording is not the ultimate purpose; the application of new technology should provide new knowledge to better understand tourist activities and optimise travel behaviour. Recently, GPS tracking technology to obtain travel spatial-temporal trajectory data has been applied in the study of tourist behaviour, but its use has been limited to description at the level of clustering and pattern analysis (De Cantis, et al., 2016; Lew \& McKercher, 2006; McKercher, et al., 2012; Shoval, et al., 2011). The accurate and constructive description of tourists' spatial-temporal behavior could help with proposed diagnosis, analysis, and countermeasure issues to better analyze what has already happened. If forestalling actions are considered, then studies about what will happen in the future become much more important. Thus, the answer to this research question is valuable for optimizing the behavior of both tourists and attractions/destinations, which are the essential areas of visitor management and destination management.

Although predicting and recommending the potential location of a newcomer based on the person's preferences and the experiences of past tourists makes sense for both tourists and tourism product suppliers (Tsai \& Chung, 2012), until recently few studies have attempted to predict tourist movement trends at the level of a tourist attraction (Xia, et al., 2009; Xia, et al., 2011). This study seeks to fill this research gap by meeting three objectives. First, we collect movement data using GPS technology to ensure high data quality. Second, we apply a grid-based clustering method to reduce data-processing complexity and retain only the most important information. Third, we design a heuristic algorithm based on data mining to predict the potential location of a focal tourist based on the person's current trajectory and the experience of previous tourists. To evaluate the performance of the proposed method, a case study was conducted at the Summer Palace in Beijing, China. We collected the movement information of 117 tourists using GPS, and obtained 111 valid trajectories. The results of an independent-samples t-test indicate that our method performed significantly better than existing methods. Furthermore, we discuss the potential applications of the proposed method, such as personalized location-based service, tourist attraction administration, and real-time crowd control.

This study will contribute by expanding the application of tourist movements in the 
mobile Internet era, in which movement data can be collected more easily; by proposing a new method that improves prediction accuracy compared with current methods; and by achieving a beneficial trade-off between prediction accuracy and efficiency.

The remainder of this paper is organised as follows. Section 2 gives an overview of the related work, which extends beyond tourism to include transportation. In Section 3, we propose several definitions and the methodological framework, after which the heuristic prediction algorithm (HPA) is described in detail. Section 4 provides the experimental results and the performance of the algorithm. Section 5 discusses the application of the prediction method in personalized location-based service, attraction/destination planning and management and real-time crowd control. We conclude the paper in Section 6 and propose a possible direction for future research.

\section{Literature review}

\subsection{Movements of tourists}

The study of this issue began with (Campbell, 1967). However, most such studies have involved mapping and modelling of movements between destinations (Mckercher \& Lau, 2008), as tourist destinations are not independent, but exist within a competing and complementary relationship (Wall, 1978). A potential tourist is likely to visit multiple destinations on a single trip to reduce the time and cost associated with travel (Ben-Akiva \& Lerman, 1985). The study by Lue, et al. (1993) supports this notion, and summarised five movement patterns. Oppermann (1995) also proposed various models for travel itineraries, two for single destinations and five for multiple destinations. Hwang, et al. (2006) examined the multicity trip patterns of international tourists in the United States, while Li, et al. (2008) revealed the spatial patterns of travel flows and travel propensity in the Asia-Pacific region over a 10-year period. Yang, et al. (2013) analysed the decisions of tourists with respect to sequential destinations using a dataset from an on-site tourist survey in Nanjing, China.

In recent years, the movement of tourists within a destination has received greater attention. Tideswell and Faulkner (1999) focused on the travel pattern of international visitors in Queensland, Australia, and explored the synergy between these patterns and destination development. Fennell (1996) studied tourist movements in the Shetland Islands using measures of space, time, perception, region and core/periphery. McKercher and colleagues did considerable exploration of tourist movements within an urban destination, using an inductive approach to identify factors that influence such movements in Hong Kong (Lew \& McKercher, 2006). They then identified 78 discrete movement patterns, which they categorised into 11 
movements styles (Mckercher \& Lau, 2008).

Compared to inter- and intra-destination movements, the study of intra-attraction movements (e.g., in national parks, protected areas, theme parks, etc.) has been inhibited by the diversity of tourist movements (Mckercher \& Lau, 2008) and the difficulties of gathering accurate location information from tourists at the micro level (Lau \& McKercher, 2006). Recently, benefiting from advances in tracking technology, e.g., GPS, GIS, RFID, APPs, etc., new studies are constantly emerging. For example, Connell and Page (2008) revealed a variety of itinerary patterns at Loch Lomond and the Trossachs National Park in Scotland through a map-based questionnaire and GIS; Orellana, et al. (2012) explored visitor movement patterns in the Dwingelderveld National Park using GPS; Smallwood, et al. (2012) analysed visitor movement patterns using travel networks in a large marine park and revealed that visitor movements were highly dependent on the road network.

These studies make up for the inadequacy of previous tourist movement research at the micro level; however, most of them have particularly emphasised qualitative factors affecting tourist movements. Xia et al. have explored this issue using models (Xia \& Arrowsmith, 2005; Xia, et al., 2009; Xia, et al., 2011), broadening perspectives on tourist movements.

\subsection{Movement prediction}

Although a number of studies have mapped and modelled tourist movements, to date few have attempted to predict trends in tourist movements. Xia, et al. (2009) analysed tourist movement data and then predicted tourist movements using Markov chains. Two years later, they proposed the semi-Markov processes that incorporate the time dimension (Xia, et al., 2011). To our knowledge, these two studies are the best attempt so far to predict intra-attraction tourist movements. Although they undertook pioneering exploration of this issue, their studies still reflect several shortcomings. First, Markov-based methods predict tourist movements on the assumption of first-order dependence, that is, that the probability distribution of the next location is independent of past locations, although many studies show that prediction accuracy based on location history is closely related to the number of historical locations available (Lu, et al., 2013; C. Song, et al., 2010; L. Song, et al., 2006). Second, tourist movement data in their study were collected via a self-administered questionnaire, which is subject to error and imprecision (Debbage, 1991; Hallo, et al., 2012). Third, location prediction based on the number of attractions the tourist will visit is impractical in real-time prediction, as the choice of routes by tourists may change over time. Because of the limited amount of research on the prediction of intra-attraction movements, and the similarity between tourist movements and human movement, it is indispensable to review the related work on human movement. 
Human movement is a complex process; however, numerous studies have revealed that human mobility shows great spatial-temporal regularity (Gonzalez, et al., 2008; Lu, et al., 2013; C. Song, et al., 2010). This fact makes it possible to predict human movement based on a person's previous history (Chen, et al., 2011). Given its importance for understanding population distribution and congestion (Batty, 1997) and urban planning (Kenett \& Portugali, 2012; Lu, et al., 2012), and for providing better location-based services (LBSs) (Chen, et al., 2010; Chen, et al., 2011; Vu, et al., 2009), both practitioners and researchers have paid considerable attention to the accurate prediction of human mobility. Over the past decades, a myriad of predictive models have been proposed, based on various methods including Markov (Dong \& He, 2007; Lin \& Yeh, 2013) and data mining (Chen, et al., 2011; Vu, et al., 2009; Yavaş, et al., 2005).

Most of these models depend heavily on prior movement history, and many have revealed that prediction accuracy is closely related to the amount of location history available ( $\mathrm{Lu}$, et al., 2013; C. Song, et al., 2010; L. Song, et al., 2006). However, it is also recognised that the processing of lengthy location histories is time consuming and computationally inefficient. Therefore, finding a trade-off between prediction accuracy and efficiency is becoming increasingly important as interest grows in making real-time location predictions. To this end, academics have increasingly explored the possibility of discarding part of the historical data. For example, the basic Markov model proposes that the probability distribution of the next location is independent of all past locations (Xia, et al., 2009; Xia, et al., 2011), but its prediction accuracy is poor because the direction of travel is not encoded when observing only the current location. Therefore, higher-order Markov models have been proposed that are sensitive to a certain number of most recent locations, yet identifying the appropriate order in a higher-order Markov model remains an open question (Ashbrook \& Starner, 2003). Aware of this issue, Chen, et al. (2011) considered the most recent historical locations on the assumption that they would have a more significant effect on route prediction than would earlier movements. However, some researchers believe that discarding some of the data will sacrifice prediction accuracy by losing valuable information (Laasonen, 2005). Moreover, the assumption that recent locations are more important for prediction than earlier locations has been questioned. Therefore, evaluating the quantity of historical information attached to each observed location and then removing those locations that provide minimal information may offer a solution. Yet, as far as we know, little attention has been directed to this issue.

To date, few studies have attempted to predict tourist movement trends at the tourist 
attraction level. The Markov-based model is one of the few methods to have been used as a predictor of tourist location; however, Markov-based methods predict tourist movements on the assumption of first-order dependence and ignore the influence of the historical position on the next position. This assumption is not practical in many cases, leading the poor prediction performance. In the transportation field, myriad predictive models have been proposed to predict human mobility. However, most of these methods have difficulty adequately resolving the trade-off between prediction accuracy and efficiency.

Therefore, this paper presents a heuristic prediction algorithm to predict the next locations of tourists using GPS data. A series of strategies are then proposed to achieve a beneficial trade-off between prediction accuracy and efficiency.

\section{Methodologies}

\subsection{Definition and framework}

In this section, we first clarify some terms and variables used in this paper. Afterwards, the framework of our method is briefly introduced.

(1) Definition

This paper draws on the fields of tourism, mathematics and data mining. For clarity, Table 1 lists the mathematical notations used and their meanings in this study.

Table 1 Mathematical notations and descriptions

\begin{tabular}{cl}
\hline Notation & \multicolumn{1}{c}{ Description } \\
\hline $\operatorname{Tr}_{i}$ & The $i^{\text {th }}$ trajectory, $i=1,2, \ldots, N$ \\
$\boldsymbol{T R} \boldsymbol{D}$ & The database of trajectories \\
$p_{i j}$ & The $j^{\text {th }}$ point in $\operatorname{Tr}_{i}, j=1,2, \ldots, n_{i}$ \\
$g_{k}$ & The $k^{\text {th }}$ cell, $k=1,2, \ldots, M$ \\
$\boldsymbol{G}$ & The set of grid cells \\
$d_{k}$ & The density of cell $g_{k}$ \\
MinPts & The density threshold \\
$g_{k}^{c}$ & The $i^{\text {th }}$ characteristic cell, $d_{k} \geqslant$ MinPts \\
$\boldsymbol{G}^{\boldsymbol{c}}$ & The set of characteristic grid cells \\
$\boldsymbol{C T r _ { i }}$ & The $i^{\text {th }}$ characteristic trajectory, $i=1,2, \ldots, N$ \\
$\boldsymbol{C T R \boldsymbol { D }}$ & The database of characteristic trajectories \\
$\boldsymbol{S}_{\boldsymbol{C T r}}$ & The set of candidate characteristic trajectories \\
\hline
\end{tabular}

A tourist movement is a sequence of time-dependent spatial points, $\left(x_{1}, y_{1}, t_{1}\right), \ldots,\left(x_{n}, y_{n}, t_{n}\right)$, where $t_{i}$ represents the timestamp of spatial point $\left(x_{i}, y_{i}\right)(1 \leq i \leq n)$. Fig. 1 shows the approximate trajectories of movements in space with respect to time. Eight points are shown in the figure, 
$p_{1}(2,1), p_{2}(1,1), p_{3}(1,3), p_{4}(2,4), p_{5}(3,5), p_{6}(3,2), p_{7}(4,4), p_{8}(5,1)$. The spatial movement trajectory $p_{1} \rightarrow p_{3} \rightarrow p_{4} \rightarrow p_{5} \rightarrow p_{7} \rightarrow p_{8}$ is represented by dotted lines with arrows, while heavy lines denote the spatial-temporal movement trajectory. Vertical lines in the trajectory indicate static motion (e.g., staying at the points), while the slashes represent movements between points. Taking this trajectory as an example, the tourist arrives at point $p_{1}$ at time $t_{1}$ and leaves this point at $t_{2}$, and so forth, finally reaching point $p_{8}$ at $t_{11}$ and departing at $t_{12}$.

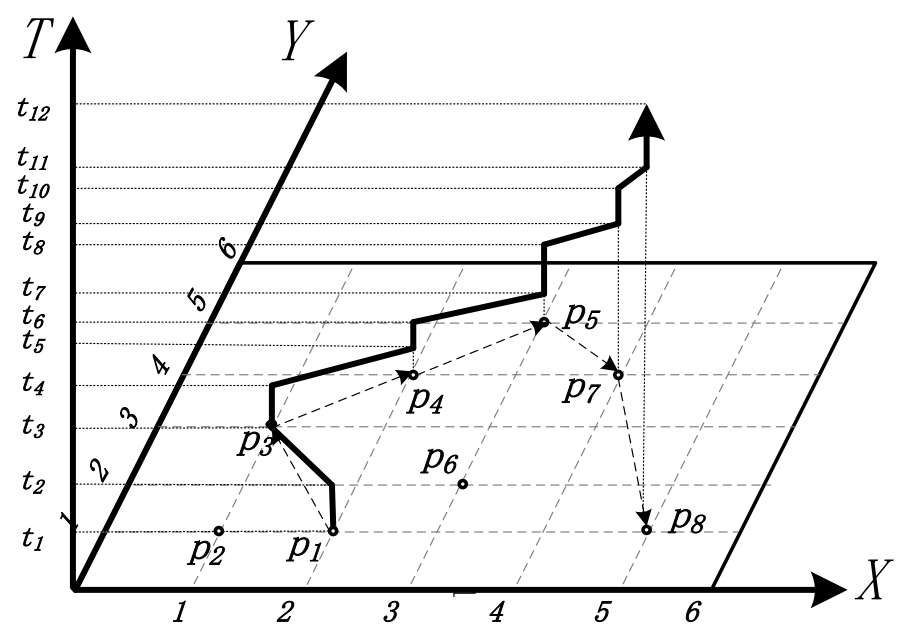

Fig. 1 The spatial-temporal movement trajectory

In this paper, we define tourist movements as a discrete process $\left\{X_{1}, X_{2}, \ldots, X_{n}\right\}$, where $\{X\}$ is the stated space. For example, a tourist trip from point $p_{1}$ to $p_{3}$ and then successively to points $p_{4}, p_{5}, p_{7}$ and $p_{8}$ can be described as the process where $X_{1}=p_{1}, X_{2}=p_{3}, X_{3}=p_{4}, X_{4}=p_{5}, X_{5}=p_{7}$, $X_{6}=p_{8}$, respectively, thus the movement trajectory can be represented as $\left\{p_{1}, p_{3}, p_{4}, p_{5}, p_{7}, p_{8}\right\}$. All of the trajectories are stored in the trajectory database (TRD).

(2) Framework

The biggest difference between the Markov-based model and our heuristic prediction algorithm (HPA) is that ours considers the influence of historical information for the next location. This problem is represented graphically in Fig. 2, where the Markov model is denoted in the blue box and the HPA in the red box. Specifically, for the Markov model, a One-Step Transition Probability Matrix is constructed according the historical trajectories stored in $\boldsymbol{T R D}$, and the next location is predicted based on the current location $g_{n}$ and the Matrix. For the HPA, the Multi-Step Transition Probability Matrix is updated not only with the current location but also with the current trajectory $\operatorname{Tr}_{0}$, which contains all of the visited locations; trajectory data mining is conducted to build this matrix. We will illustrate the details of the HPA in the following sections. 


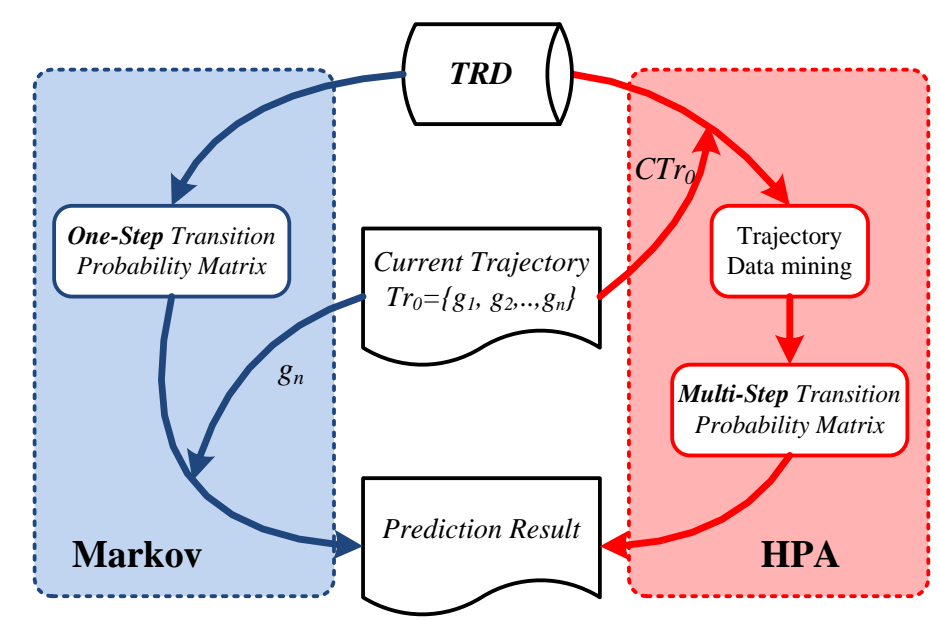

Fig. 2 The difference between the Markov model and the HPA

The methodology presented consists mainly of data preparation, tourist movement mining and tourist movement prediction. The overall framework is illustrated in Fig. 3.

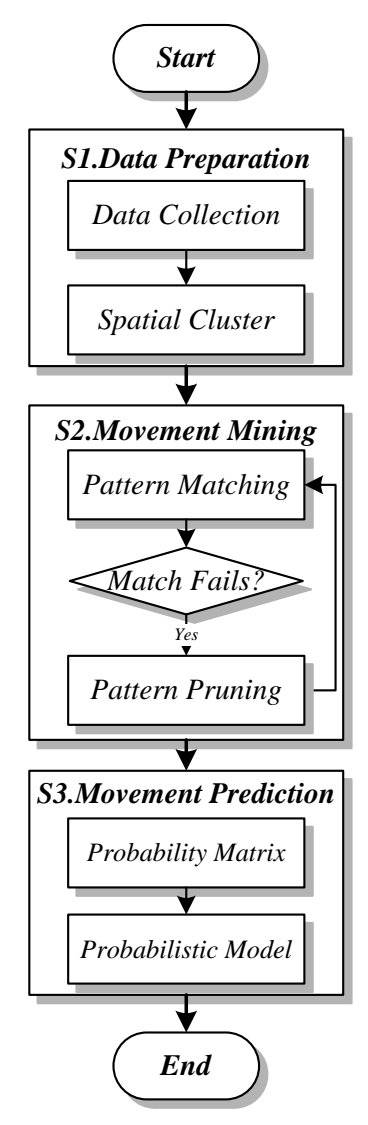

Fig. 3 The methodological framework

In the data preparation stage, the movement data of tourists within a tourist attraction is collected using GPS tracking technology. As analysing the vast amount of GPS data is cumbersome, a grid-based spatial clustering method is adopted. Movement mining is the core of the methodology and the foundation of movement prediction. At this stage, the trajectory of the current tourist is matched with the trajectories in the characteristic trajectory database CTRD using a pattern-matching algorithm. If the match fails, a movement pattern pruning 
method is presented to improve the algorithm's flexibility. In the movement prediction stage, we build a probability matrix for the next location based on the results of pattern mining, after which future movements are predicted according to the probabilistic model. We describe these three stages in detail in sections 3.2, 3.3 and 3.4, respectively.

\subsection{Module 1: Data preparation}

The accurate collection of the movement data of tourists in an outdoor environment without affecting their normal behaviour is challenging because of the potentially large number of tourists and their unconstrained behavioural (O'Connor, et al., 2005). However, as GPS tracking technology can potentially alleviate many of the problems of traditional tracking methods (Hallo, et al., 2012), more recent studies of visitor movements at tourist attractions have used GPS (Bauder, 2014; Hallo, et al., 2012; Orellana, et al., 2012; Pettersson \& Zillinger, 2011). The recorded data includes longitude, latitude, timestamp, speed, direction and so forth.

For clarity, we illustrate the data preparation with an example. As the GPS device collects a tourist's movement information, a series of GPS points is recorded. Fig. 4(a) shows the GPS points collected from all of the tourists. However, we can only detect the trajectory of a specific tourist when the GPS device tag and the timestamp are extracted from this data. One such tourist trajectory is denoted by the blue line in Fig. 5(a), which contains 23 recorded GPS points, that is, $\operatorname{Tr}_{i}=\left\{p_{1}, p_{2}, p_{3}, \ldots, p_{23}\right\}$.

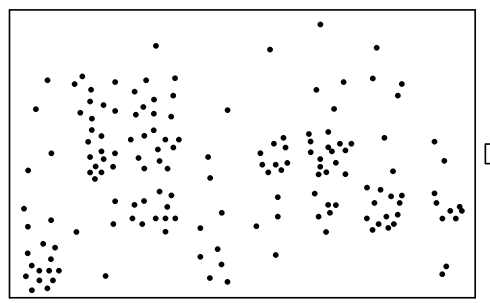

(a)

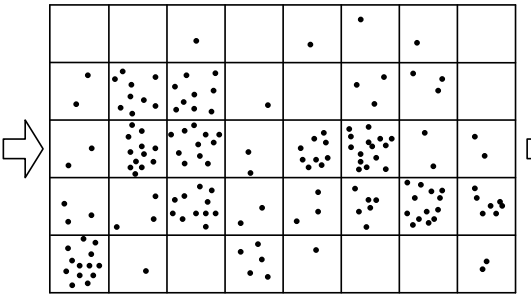

(b)

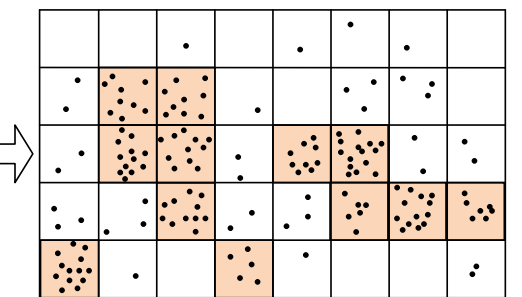

(c)

Fig. 4 Example of GPS points

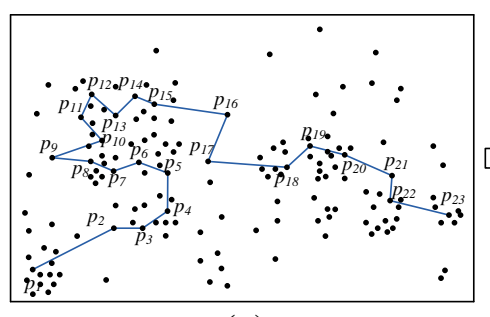

(a)

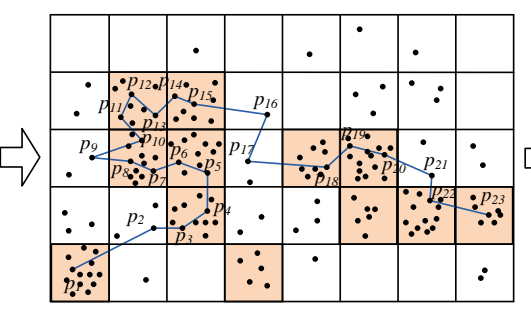

(b)

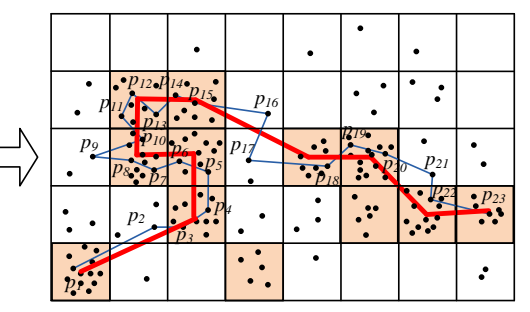

(c)

Fig. 5 Example of GPS Trajectories

However, because a huge number of points are recorded for tourists during their visit, and analysing this volume of data is cumbersome (Orellana, et al., 2012), some studies have attempted to abstract the points of interest (POIs) or characteristic points through GPS data 
clustering.

In this paper, a grid-based spatial clustering method is adopted. First, the study area is divided into several axa grid cells. For example, the study area in Fig. 4 is divided into 40 cells, as shown in Fig. 4(b), $G=\left\{g_{1}, g_{2}, \ldots, g_{40}\right\}$. The parameter density $d_{k}$ expresses the number of GPS points in cell $g_{k}$. Supposing that $p_{i j}$ is the $j$ th point in $T r_{i}$, if $p_{i j}$ is contained in $g_{k}$, that is $p_{i j} \in g_{k}$, set 0-1 discrete variable $x_{i j k}=1$; otherwise $x_{i j k}=0$. As each point belongs only to one cell, that is: $\sum x_{i j k}=1, k=1,2, \ldots, M$. The density of a cell can be calculated using Eq.(3.1):

$$
d_{k}=\sum_{i=1}^{N} \sum_{j=1}^{n_{i}} x_{i j k}
$$

The density threshold is specified by choosing the minimum number of points MinPts in a cell, that is, if $d_{k} \geqslant$ MinPts, then $g_{k}$ is a characteristic cell denoted $g_{k}^{c}$. If we set MinPts $=5$, there are 12 characteristic cells, as in Fig. $4(\mathrm{~b}), G^{c}=\left\{g_{1}^{c}, g_{8}^{c}, \cdots, g_{37}^{c}\right\}$, shown as shaded cells in Fig. 4(c). We keep only the characteristic cells and discard the others, which is reasonable as the more points a cell contains, the more information the cell carries. The original trajectory $\left\{p_{1}, p_{2}\right.$, $\left.p_{3}, \ldots p_{23}\right\}$ can then be denoted as a characteristic trajectory $C T r_{i}=\left\{g_{1}^{c}, g_{12}^{c}, g_{12}^{c}, \cdots, g_{37}^{c}\right\}$, because $p_{1} \in g_{1}^{c}, \quad p_{3}, p_{4} \in g_{12}^{c}, \ldots, \quad p_{23} \in g_{37}^{c}$. The characteristic trajectory is denoted using the red line in Fig. 5(c). All of the trajectories in $\boldsymbol{T R D}$ are converted to characteristic trajectories and stored in the $C T R D$.

It should be noted that this simplification is expected to increase prediction efficiency because of the reduction in data processing. However, removing partial history is tied to a concern about the loss of valuable information, which makes predictions less accurate. Therefore, selecting an appropriate MinPts value is crucial for achieving a beneficial trade-off between prediction accuracy and efficiency. Sensitivity tests for the performance of the MinPts value in movement prediction are presented in the case study.

\subsection{Module 2: Movement mining}

For a clearer illustration, we will delimit several definitions used in this paper.

Definition 1 (Subset): For two characteristic trajectories $C T r_{i}$ and $C T r_{j}$, if any cell in $C T r_{i}$ is also contained in $C T r_{j}$, that is, $\forall g_{k}^{c} \in C T r_{i}: g_{k}^{c} \in C T r_{j}$, then: $C \operatorname{Tr}_{i}$ is a subset of $C \operatorname{Tr}_{j}$, denoted as $C \operatorname{Tr}_{i} \subseteq C T r_{j}$.

Definition 2 (Same Trajectory): For two characteristic trajectories $C \operatorname{Tr}_{i}$ and $C \operatorname{Tr}_{j}$, suppose $C T r_{i} \subseteq C \operatorname{Tr}_{j}$ and $C T r_{i} \supseteq C \operatorname{Tr}_{j}, g_{i k}^{c}$ and $g_{j k}^{c}$ are the $k^{\text {th }}$ cell in $C \operatorname{Tr}_{i}$ and $C \operatorname{Tr}_{j}$, respectively. For any $k \leq n_{i}=n_{j}$, if the equation $g_{i k}^{c}=g_{j k}^{c}$ holds, then $C \operatorname{Tr}_{i}$ is the same as $C \operatorname{Tr}_{j}$. That is $\forall k \leq n_{i}=n_{j}: g_{i k}^{c}=g_{j k}^{c}$, then $C \operatorname{Tr}_{i}=C \operatorname{Tr}_{j}$, where $n_{i}$ and $n_{j}$ are the number of characteristic grid 
cells contained in $C T r_{i}$ and $C T r_{j}$, respectively.

Definition 3 (Sub-Trajectory): Suppose $C \operatorname{Tr}_{i} \subseteq C \operatorname{Tr}_{j}$, and $\operatorname{SubTr}\left[C \operatorname{Tr}_{i}, x, y\right]$ reports just a section of the given characteristic trajectory $\mathrm{CTr}_{i}$, ranging between the first position $x$ and the second position $y$. If there is any integer $k \leq n_{j}-n_{i}$, such that Eq.(3.2) holds, then $C T r_{i}$ is the sub-trajectory of $C \operatorname{Tr}_{j}$, denoted as $C \operatorname{Tr}_{i} € C \operatorname{Tr}_{j}$.

$$
C \operatorname{Tr}_{i}=\operatorname{SubTr}\left[C \operatorname{Tr}_{j}, k, k+n_{i}\right], k \leq n_{j}-n_{i}
$$

In this section, a pattern-mining algorithm is proposed to mine the movement pattern based on the current trajectory of a focal tourist and the CTRD. We describe how to mine movement patterns based on the definitions in the pseudo code shown in Fig. 6. Given the characteristic trajectory database $(\boldsymbol{C T R D})$ and current characteristic trajectory $C \operatorname{Tr}_{0}$, the output of our algorithm is the candidate set $\boldsymbol{S}_{\boldsymbol{C} T r}$ (line 1-2 in Fig. 6). Initially, the $C \operatorname{Tr}_{0}$ is used as the input sequence (IS) to match the candidate characteristic trajectories (line 3 in Fig. 6). The whole process of pattern mining is shown from lines 4-16 in Fig. 6. The $I S$ is matched with each characteristic trajectory in $C T R D$, if $I S E C \operatorname{Tr}_{i}\left(C T r_{i} \in C T R D\right)$, then $C \operatorname{Tr}_{i}$ is entered into $\boldsymbol{S}_{\boldsymbol{C T r}}$. The procedure of movement-pattern mining is shown in lines 5-9 in Fig. 6. If the match fails, that is $S_{C T r}=\varnothing$, then a movement pattern pruning method is presented to improve the flexibility of the algorithm. Movement pattern pruning is of particular significance to movement-pattern mining. However, other than the Chen, et al. (2011) study, discussion of this issue in the research is negligible. In addition, although Chen, et al. (2011) provide a comprehensive method, it is still based on the assumption that a recent location is more important than an earlier one. Our work seeks to avoid this assumption and rather remove the cells with less information $\left(g_{k}^{c}\right.$ with minimum density); see lines 13-14 in Fig. 6.

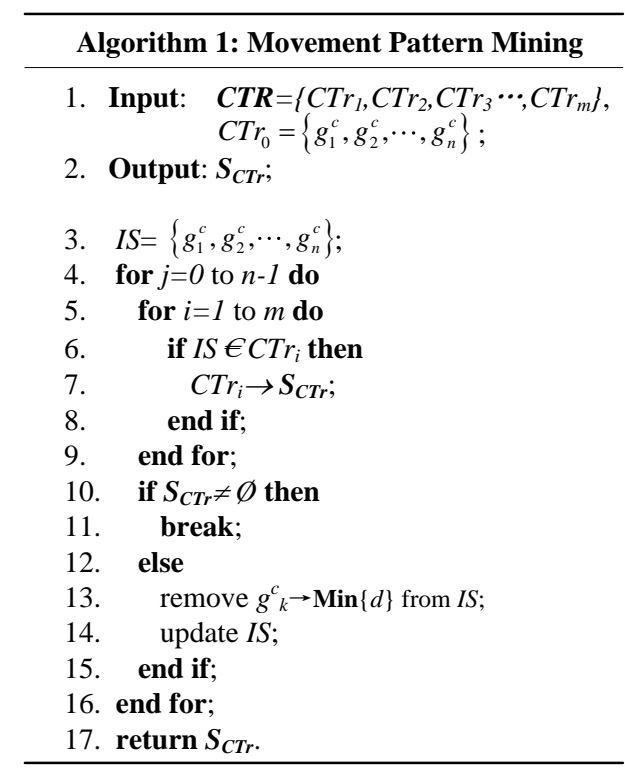

Fig. 6 The algorithm for movement-pattern mining 


\subsection{Module 3: Movement prediction}

In this section, we build the movement prediction model based on a probabilistic analysis. Given the current trajectory of the focal tourist $C \operatorname{Tr}_{0}=\left\{g_{1}^{c}, g_{2}^{c}, \cdots, g_{n}^{c}\right\}$, and its candidate set $S_{C T r}$, the prediction of the next cell the tourist will visit can be determined by the joint probability, as shown in Eq.(3.3):

$$
P\left(g_{k}^{c}, C T r_{0}\right)=P\left(g_{k}^{c} \mid C T r_{0}\right) \cdot P\left(C T r_{0}\right)
$$

Where $P\left(g_{k}^{c}, C T r_{0}\right)$ is the probability that $g_{k}^{c}$ and $C T r_{0}$ simultaneously occur-that is, the probability that a tourist will visit cell $g_{k}^{c}$ right after the current trajectory $C T r_{0}$. $P\left(g_{k}^{c} \mid C T r_{0}\right)$ is the conditional probability that cell $g_{k}^{c}$ will be visited given $C T r_{0}$. Because the tourist is currently visiting the trajectory $C T r_{0}$, that is, $P\left(C T r_{0}\right)=1$, $P\left(g_{k}^{c}, C T r\right)=P\left(g_{k}^{c} \mid C T r_{0}\right)$. The difference between our prediction algorithm and the Markov-based model (Xia, et al., 2009; Xia, et al., 2011) is that the probability matrix is updated not only with the current location but also with the current trajectory, which contains all of the visited cells. Therefore, the probability matrix of the next cell is $M\left(C T r_{0}, S_{C T r}\right)$ (shown as Eq. (3.4)), where $C \operatorname{Tr}_{0}$ is the current trajectory of the focal tourist and $S_{C T r}$ is the set of candidate characteristic trajectories resulting from the movement pattern mining.

$$
M\left(C \operatorname{Tr}_{0}, S_{C T r}\right)=\left[\begin{array}{cccc}
P\left(g_{1}^{c} \mid C \operatorname{Tr}_{0}, C T r_{1}\right) & P\left(g_{2}^{c} \mid C T r_{0}, C \operatorname{Tr}_{1}\right) & \cdots & P\left(g_{M}^{c} \mid C T r_{0}, C \operatorname{Tr}_{1}\right) \\
P\left(g_{1}^{c} \mid C T r_{0}, C \operatorname{Tr}_{2}\right) & P\left(g_{2}^{c} \mid C T r_{0}, C T r_{2}\right) & \cdots & P\left(g_{M}^{c} \mid C \operatorname{Tr}_{0}, C \operatorname{Tr}_{2}\right) \\
\cdots & \cdots & \cdots & \ldots \\
P\left(g_{1}^{c} \mid C T r_{0}, C T r_{w}\right) & P\left(g_{2}^{c} \mid C T r_{0}, C T r_{w}\right) & \cdots & P\left(g_{M}^{c} \mid C T r_{0}, C T r_{w}\right)
\end{array}\right]
$$

Where the conditional probability $P\left(g_{k}^{c} \mid C T r_{0}, C T r_{i}\right)$ is the probability of visiting cell $g^{c}$ right after the current trajectory $C T r_{0}=\left\{g_{1}^{c}, g_{2}^{c}, \cdots, g_{n}^{c}\right\}$ along the movement pattern $C T r_{i}$, and $C T r_{i} \in S_{C T r} ; w$ is the number of candidate characteristic trajectories in $S_{C T r}$. The probability of visiting cell $g_{k}^{c}$ depends on the sum of $P\left(g_{k}^{c} \mid C T r_{0}, C T r_{i}\right)$, which can be calculated using Eq. (3.5). Furthermore, the probability matrix $M\left(C T r_{0}, S_{C T r}\right)$ can be simplified as Eq.(3.6) through the accumulation of column vectors according to Eq.(3.5). Finally, the next location of the focal tourist is predicted with the highest probability in $M\left(C \operatorname{Tr}_{0}, S_{C T r}\right)$.

$$
\begin{gathered}
P\left(g_{k}^{c}, C T r_{0}\right)=\sum_{i=1}^{w} P\left(g_{k}^{c} \mid C T r_{0}, C T r_{i}\right), C T r_{i} \in S_{C T r} \\
M\left(C T r_{0}, S_{C T r}\right)=\left[P\left(g_{1}^{c}, C T r_{0}\right) P\left(g_{2}^{c}, C T r_{0}\right) \cdots P\left(g_{M}^{c}, C T r_{0}\right)\right] \\
=\left[\begin{array}{llll}
\sum_{i=1}^{w} P\left(g_{1}^{c} \mid C T r_{0}, C T r_{i}\right) & \sum_{i=1}^{w} P\left(g_{2}^{c} \mid C T r_{0}, C T r_{i}\right) & \cdots & \sum_{i=1}^{w} P\left(g_{M}^{c} \mid C T r_{0}, C T r_{i}\right)
\end{array}\right]
\end{gathered}
$$




\section{Performance evaluation and discussion}

\subsection{Case study area}

The aim of this study was to build an approach to predict the next location decision of tourists based on their GPS tracking data, thus, the complexity of the spatial structure of attractions was considered when choosing the case site. The Summer Palace in Beijing, China, remains one of the nation's most popular tourist attractions, a map of which is shown in Fig. 7. In December 1998, UNESCO included the Summer Palace on its World Heritage Site list, bringing it to the international tourist market. The landscape of the Summer Palace is dominated by Longevity Hill and Kunming Lake, and covers $2.9 \mathrm{~km}^{2}$, three quarters of which is under water. Its $70,000 \mathrm{~m}^{2}$ of building space features a variety of palaces, gardens and other ancient architectural structures. The Summer Palace encompasses more than 100 tourist spots and comprises three areas with different functions and themes, namely the administrative, residential and scenic areas (Editorial Committee of Beijing Chorography, 2004).

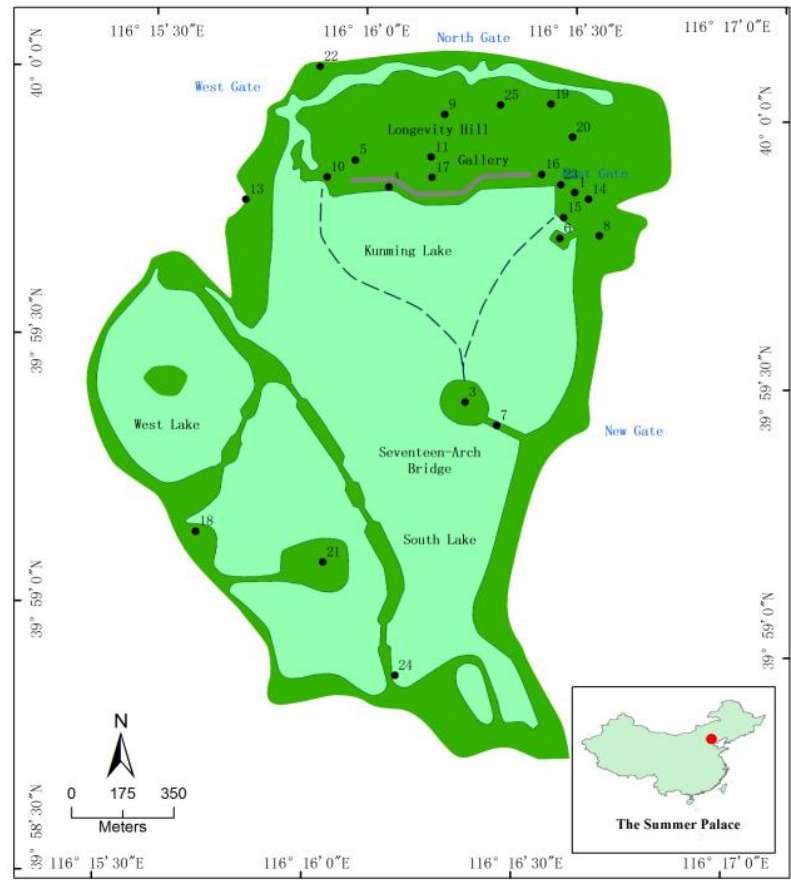

Fig. 7 Summer Palace map*

\subsection{Data preparation for the Summer Palace}

The survey of spatial-temporal tourist behaviour at the Summer Palace took place during

\footnotetext{
* 1-Garden of Virtue and Harmony; 2-Garden of Harmonious Pleasures; 3-South Lake Island; 4-Long Corridor; 5-Strolling through a Picture Scroll; 6-Heralding Spring Pavilion; 7-Seventeen-Arch Bridge; 8-Wenchang Gallery; 9-Four Great Regions; 10-Stone Archway; 11-Tower of the Fragrance of the Buddha; 12-Suzhou Street; 13-Scenic Area of 'Pictures of Farming and Weaving'; 14-Hall of Benevolence and Longevity; 15-Hall of Jade Ripples; 16-Hall of Happiness in Longevity; 17-Hall that Dispels the Clouds; 18-Hall of Good Sight; 19-Hall of Serenity; 20-Hall of Utmost Blessing; 21-Hall of Zaojian; 22-Hall for Listening to Orioles; 23-Yiyun Hall; 24-Causeway of Kuming Lake; 25-Tower of Duobao
} 
12-13 December 2010. The survey group captured data on tourists' intra-attraction spatial-temporal behavior and demographic characteristics using handheld GPS tracking devices and activity diary questionnaires. To keep the sample representative and avoid sample bias, random sampling was used in this survey. To track complete behavior paths, GPS devices were given to volunteers at every entrance of the Summer Palace and collected at the exits when they left. The researchers did not choose the respondents subjectively; they were instructed to stand at the entrances to the Summer Palace with GPS devices and questionnaires ready and to invite the first visitor they met to participate in the survey. If the visitor refused, they were to ask the next one until they found a willing tourist. Tourists who were willing to participate were asked to take the GPS device and then to complete the questionnaire upon returning the device at the end of their visit. All of the space-time paths of the respondents were recorded by the server. The GPS devices automatically recorded the latitude and longitude of tracking points every three minutes with positioning time, instantaneous velocity, and direction information, while the error is less than 10 meters.

The questionnaire contained five parts: personal information (gender, nationality, age, education, occupation and monthly income), consumption information (entrance fee, guide map, boat ticket, self-guided machine, refreshments, souvenirs and guide services), sites visited and activity choices, visit information (companions, transport, motivation and frequency) and route information. Route information was collected using the guide map of the Summer Palace. Respondents were asked to draw their route on the map from the gate where they entered to the gate where they exited, number each site in the sequence visited, and fill in a table to show the time of entry and exit at each site in sequence. The investigators only approached tourists who had just finished their visit to the Summer Palace. While administering the questionnaire, pictures of the Summer Palace sites were used as tips to refresh respondents' memories of the appearance and name of the various sites.

During this survey, GPS devices were delivered 117 times, with only one per group. When the respondents returned their GPS devices, they were asked to fill in a questionnaire with demographic and travel experience information. A sample was considered valid only when both the GPS trajectory and a matching questionnaire were completed. The validity rate was $94.9 \%$, and the GPS trajectory, demographic and travel data for 111 respondents was used in this analysis. Of the respondents, $53.0 \%$ were male, $30.2 \%$ were first-time visitors to the Summer Palace, and $68.8 \%$ had a high school degree or above.

GPS technology offers a number of advantages over traditional methods for understanding tourist mobility within an attraction (Hallo, et al., 2012); however, some errors 
are inevitable. For example, the GPS can report an obviously incorrect location point. We resolve these errors by excluding these points using the information concerning the time at which the points were reported (Pettersson \& Zillinger, 2011). After excluding these points, 5,867 GPS points collected from 111 tourists remained, as shown in Fig. 8(a). We divided the study area into 2,000 grid cells of $50 \mathrm{~m} \times 50 \mathrm{~m}$ and calculated the density of each cell based on Eq.(3.1). The density distribution is shown in Fig. 8(b).

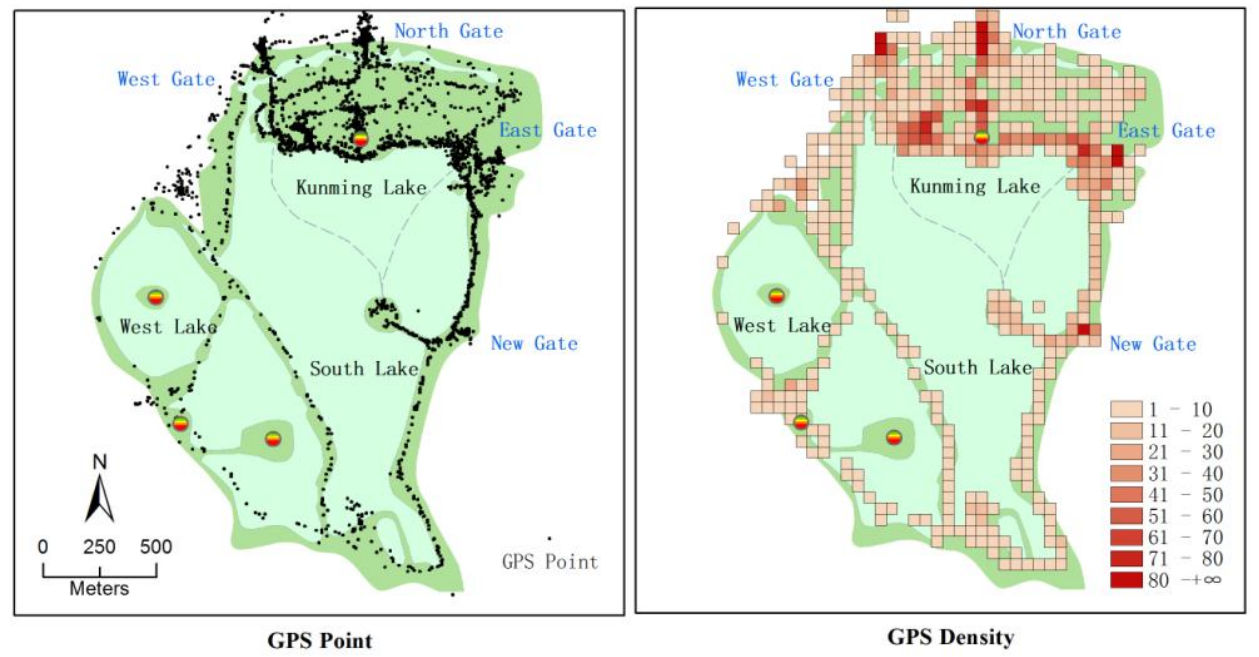

Fig. 8 The GPS points and grid cells

As mentioned above, the MinPts value determines how many characteristic cells and GPS points will be discarded; this in turn influences the loss of information. Therefore, it is necessary to identify the relationships between the number of characteristic grid cells, the number of GPS points contained in the characteristic grid cells, and the value of the parameter MinPts, as shown in Fig. 9. As seen in Fig. 9, both the characteristic cells (denoted by heavy blue lines) and GPS points (green dotted lines) fall dramatically where MinPts $\leq 50$, after which they slowly but steadily decrease until MinPts $=293$. In fact, only $21.6 \%(432$ of 2,000) of the cells were visited by tourists, that is $d_{k}>0$; while about $1 \%(21$ of 2,000$)$ of the cells contain more than 50 GPS points, that is $d_{k}>50$.

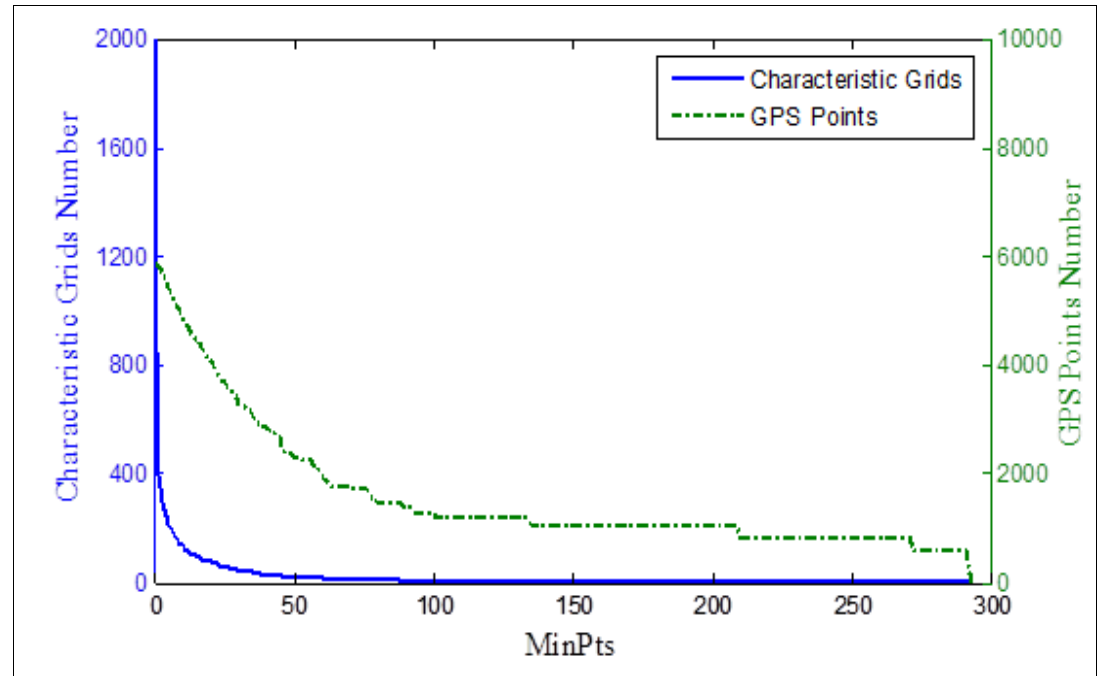

Fig. 9 Relationship between Characteristic Grid cells and MinPts 


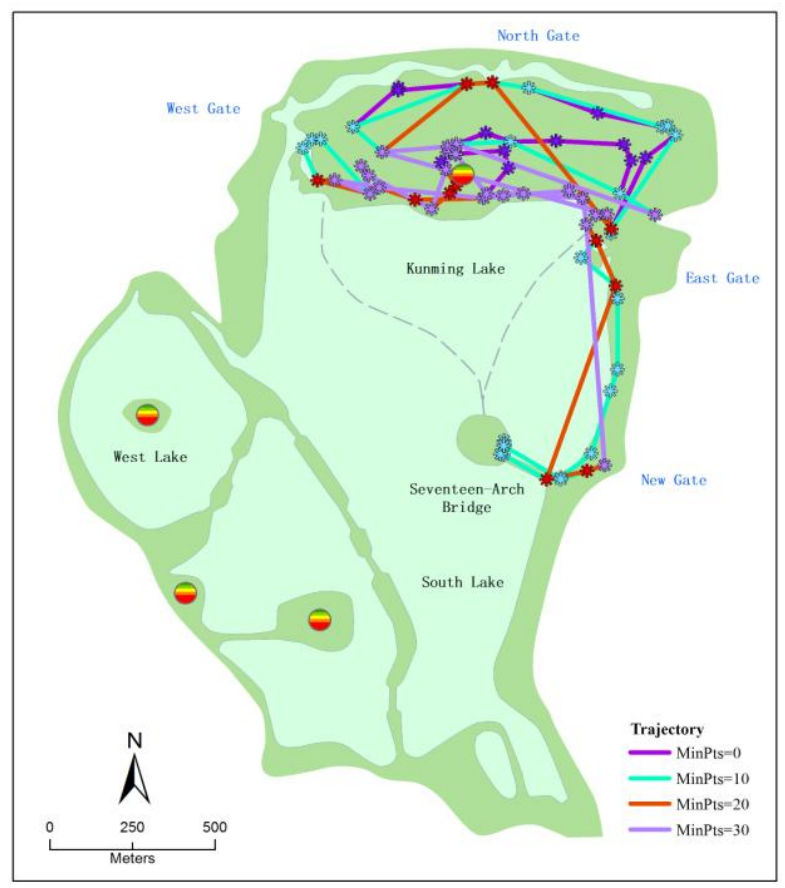

Fig. 10 The example of 4 characteristic trajectories

According to Section 3.2, the characteristic trajectories can be represented as a sequence of characteristic grid cells. Thus, the characteristic trajectories depend on the value of MinPts. To illustrate this, we draw four characteristic trajectories for the 11th tourist when MinPts=0, 10, 20, and 30, respectively, as shown in Fig. 10. The characteristic trajectory retains all of the movement information when MinPts=0; as the value of MinPts increases, the grid cells with $d_{k}<$ MinPts are removed from the trajectory. Therefore, the greater the MinPts value, the fewer grid cells the characteristic trajectory includes. For example, the number of characteristic grid cells in these four trajectories is $141,118,85$ and 61 , respectively. Consequently, selecting an appropriate MinPts value is crucial to reduce data processing complexity while retaining the most important information. Sensitivity tests for the performance of the MinPts value in movement prediction are presented in Section 4.3.

\subsection{Movement prediction in Summer Palace}

This section has two purposes: first, to evaluate the performance of our method in comparison to other methods, and second, to test how the parameter MinPts affects performance.

For the performance evaluation, it is necessary to illustrate the experiment design process and the index that reflects the performance. For the experiment, we select 20 of the 111 trajectories using the equal probability selection method; the remaining trajectories become the training dataset. We then divide the test trajectories into two sections. The latter section is hidden for test purposes, and the former section is set as the current trajectory, which 
predicts the place the tourist will visit in the subsequent trajectory. Prediction accuracy is introduced to evaluate the performance, and is denoted as follows: if the selected tourist 1 chooses the next location as its prediction, the prediction is correct; in this case, we set the 0-1 discrete variable to $\pi_{l}=1$ and to $\pi_{l}=0$ otherwise. Then, the prediction accuracy in an experiment is defined as $\sum \pi / L_{s}\left(l=1,2, \ldots, L_{s}\right)$, where $L_{s}$ is the number of test trajectories.

For the first purpose of comparing methods, the Markov-based model proposed by Xia et al.(2009; 2011) is used as a baseline. We set the parameter MinPts=40, resulting in 34 characteristic grid cells, while the number of GPS points contained in these characteristic grid cells is 2,878 . To reduce the interference of random factors, we repeat each experiment 100 times. The experimental results are presented graphically in Fig. 11.

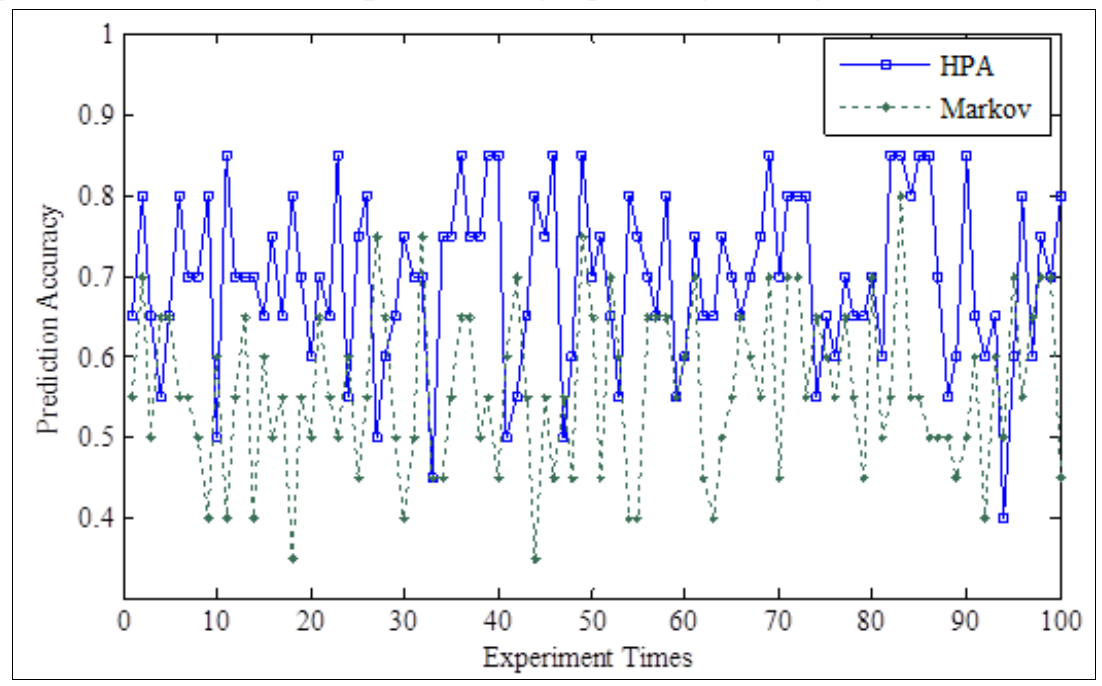

Fig. 11 Experimental results of the two methods

To further analyze the performance of these two methods, an independent sample $t$-test is conducted. The mean and standard deviation of the prediction accuracy for these two methods are displayed in Table 2. Table 3 shows the results of the independent sample $t$-test (Levene's test for equality of variances, $F=0.033, p=0.855)$. The results indicate that the method proposed in this paper achieves significantly greater accuracy $(M=0.6975, \mathrm{SD}=0.10454)$ than the Markov-based method $(M=0.5595, \mathrm{SD}=0.10118)(t(100)=9.486, \mathrm{p}<0.05)$. Similarly, the $t$-test results for MinPts $=0,10,20,30$, and 50 listed in Table 4 also show significantly better accuracy for our proposed method than for the Markov-based method.

Table 2 Group Statistics $($ MinPts=40)

\begin{tabular}{llcccc}
\hline & Method & $\mathrm{N}$ & Mean & Std. Deviation & Std. Error Mean \\
\hline Accuracy & HPA & 100 & .6975 & .10454 & .01045 \\
& Markov & 100 & .5595 & .10118 & .01012 \\
\hline
\end{tabular}


Table 3 Independent Samples Test $($ MinPts $=40)$

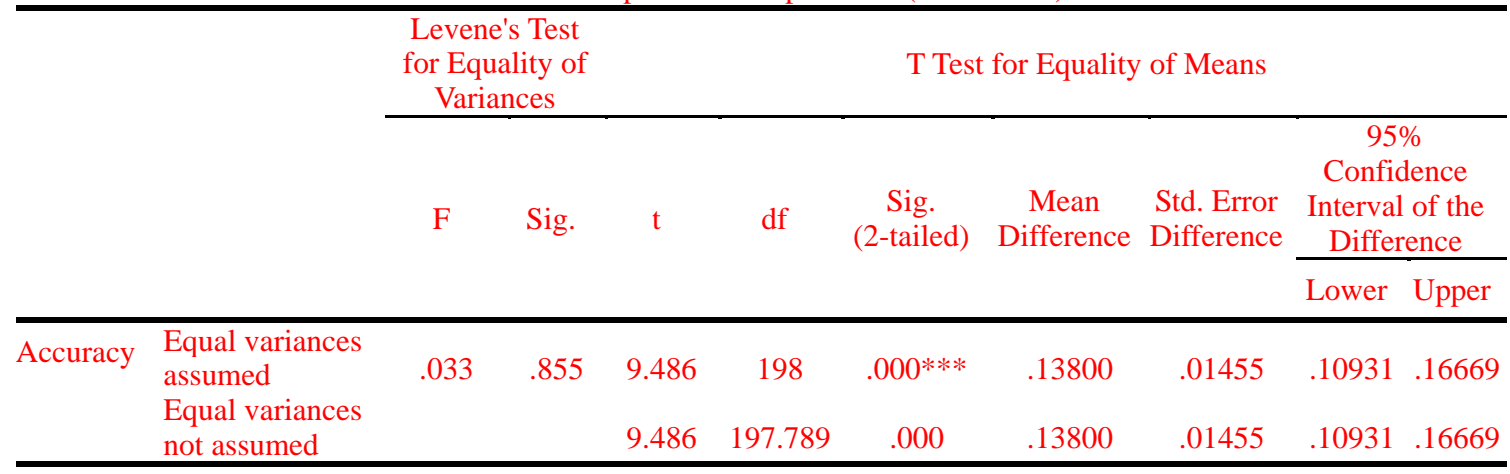

$* \mathrm{p}<0.05 ; * * \mathrm{p}<0.01 ; * * * \mathrm{p}<0.001$

Table 4 Independent Samples T Test

\begin{tabular}{ccccccc}
\hline & \multicolumn{2}{c}{ Mean } & \multicolumn{2}{c}{ SD } & \multirow{2}{*}{$\mathrm{t}$} & $\mathrm{p}$ \\
\cline { 2 - 5 } & HPA & Markov & HPA & Markov & & \\
\hline MinPts $=0$ & 0.4365 & 0.32 & 0.1220 & 0.1015 & 7.340 & $.000^{* * * *}$ \\
MinPts $=10$ & 0.4535 & 0.3395 & 0.0975 & 0.1045 & 7.975 & $.000^{* * *}$ \\
MinPts $=20$ & 0.4695 & 0.3545 & 0.1152 & 0.1215 & 6.869 & $.000^{* * *}$ \\
MinPts=30 & 0.7095 & 0.5415 & 0.0971 & 0.1069 & 11.636 & $.000^{* * *}$ \\
MinPts=40 & 0.6975 & 0.5595 & 0.1045 & 0.1012 & 9.486 & $.000^{* * *}$ \\
MinPts $=50$ & 0.7245 & 0.6090 & 0.1079 & 0.1090 & 7.53 & $.000^{* * *}$ \\
\hline
\end{tabular}

$* \mathrm{p}<0.05 ; * \mathrm{p}<0.01 ; * * * \mathrm{p}<0.001$.

In the second part of this section, we test how the value of the parameter MinPts affects the performance of the proposed method by setting MinPts=0, 10, 20, 30, 40 and 50, respectively. Again, we conduct each experiment 100 times to reduce the interference of random factors. To discover any differences, an analysis of variance (ANOVA) was conducted. The descriptive statistics are listed in Table 5. The results indicate that there are statistically significant differences between groups for prediction accuracy $(F=172.643, p<$ 0.05 in Table 6). To further determine which specific groups were different, a Scheffe test was conducted (see Table 6). The accuracy achieved by the latter three groups (MinPts $=30,40$, and 50) is significantly greater than that achieved by the former (MinPts $=0,10$, and 20).

Table 5 Descriptive statistics

\begin{tabular}{lccc}
\hline & N & Mean & Std.Deviation \\
\hline MinPts=0 & 100 & .4365 & .12203 \\
MinPts $=10$ & 100 & .4535 & .09751 \\
MinPts=20 & 100 & .4695 & .11523 \\
MinPts=30 & 100 & .7095 & .09710 \\
MinPts=40 & 100 & .6975 & .10454 \\
MinPts=50 & 100 & .7245 & .10789 \\
Total & 600 & .5818 & .16808 \\
\hline
\end{tabular}


Table 6 ANOVA Results and multiple Comparisons for different Minpts

\begin{tabular}{lrrrrrr}
\hline & $\begin{array}{c}\text { Sum of } \\
\text { Squares }\end{array}$ & df & $\begin{array}{c}\text { Mean } \\
\text { Square }\end{array}$ & F & Sig. & Scheffe $(\mathrm{p}<0.05)$ \\
\hline Between Groups & 10.024 & 5 & 2.005 & $172.643^{* * * *}$ & .000 & Minpts $=30>$ Minpts $=0,10,20$ \\
Within Groups & 6.898 & 594 & .012 & & & Minpts $=40>$ Minpts $=0,10,20$ \\
Total & 16.922 & 599 & & & & Minpts $=50>$ Minpts $=0,10,20$ \\
\hline
\end{tabular}

$* \mathrm{p}<0.05 ; * * \mathrm{p}<0.01 ; * * * \mathrm{p}<0.001$

\subsection{Tourist distribution prediction at the Summer Palace}

We discussed above how to predict the location of an individual tourist. In fact, the spatial distribution of tourists within the attraction can be predicted according to the potential location of an individual. Estimations of tourist distributions are extremely important, especially for crowd control and the layout of service facilities (e.g., toilets, benches, cafes, etc.) (Hallo, et al., 2012). In this section, we discuss how to predict the spatial distribution of tourists based on the location prediction of an individual.

Suppose that the predicted grid cell of tourist $l$ at moment $t$ is defined as $\widehat{g}_{l t}$. If the predicted grid is $\widehat{g}_{k}$, that is, $\widehat{g}_{l t}=\widehat{g}_{k}$, set the $0-1$ discrete variable to $y_{l t}^{k}=1$; otherwise, set it to $y_{l t}^{k}=0$. The variable $\hat{z}_{t}^{k}$ expresses the number of tourists whose predicted grid is $\hat{g}_{k}$ at moment $t$; it can be calculated using Eq. (4.1), where $L$ is the total number of tourists.

$$
\widehat{z}_{t}^{k}=\sum_{l=1}^{L} y_{l t}^{k}
$$

The spatial distribution of tourists is reflected by the number of tourists in each grid cell. We use an $M$-dimensional vector to represent the tourist distribution, shown as Eq. (4.2), where $M$ is the total number of grid cells.

$$
D V_{t}=\left\{\widehat{z}_{t}^{1}, \widehat{z}_{t}^{2}, \cdots, \widehat{z}_{t}^{M}\right\}
$$

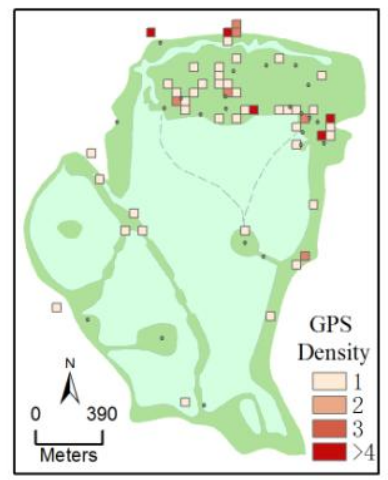

11:00

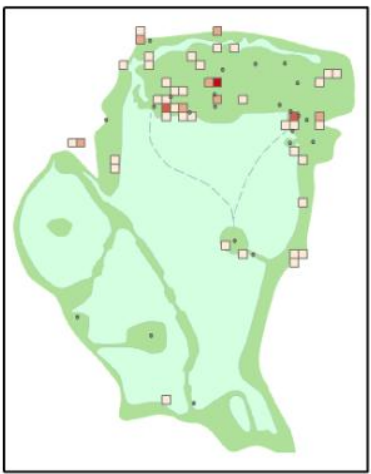

$11: 30$

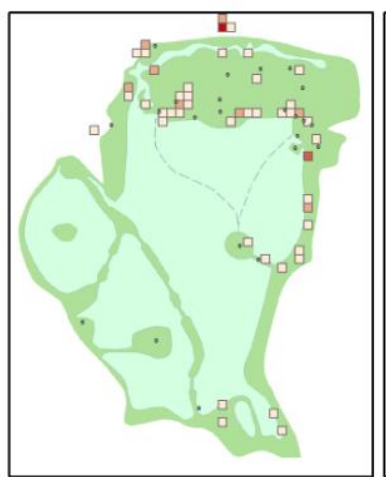

12:00

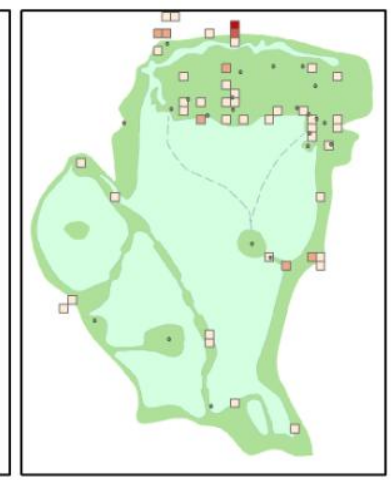

$12: 30$

Fig. 12 Tourist distribution prediction at the Summer Palace

We illustrate this by taking the 111 GPS trajectories at the Summer Palace as an example. We predict the potential spatial distribution of tourists at 11:00, 11:30, 12:00, and 12:30. The 
prediction result is represented graphically in Fig. 12, in which darker colors indicate larger numbers of tourists.

\section{Discussion}

With the ability to forecast the next location of individual tourists using HPA, a great deal of valuable information can be obtained through data mining. This information can be applied in a personalized location-based service, using the prediction results for individual tourists and in attraction/destination planning and management and for real-time crowd control using the prediction results for tourist flow.

\subsection{Personalized location-based service}

The heuristic prediction algorithm (HPA) predicts individual tourist paths, and it thus may support location-based service (LBS) with user location prediction to provide operator and reservation services to tourists. Personalized tour guide service apps could be designed with the application of the HPA; these would require users to provide their demographic information when they register. According to the GPS data for tourists at the Summer Palace, 100 tourists visited Spot 13 (shown in Fig. 7) at least once (87.3\% of male respondents and $90.9 \%$ of female respondents visited this spot). After that, 45 tourists (45\%) went to Spot 22 immediately after Spot 13. However, of the tourists who visited Spot 4, Spot 5, and Spot 13 successively, only $20 \%$ went to Spot 22 next. If visitors are willing to provide certain information, they can be provided with information and recommendations along their path with optional spots or attractions to go next rather receiving the information for the entire attraction or destination. This could be helpful in eliminating information redundancy and interference. Navigation services have become quite mature in terms of general transportation when travelers know their origin and destination. However, in tourist navigation, tourists are in the process of deciding where to go next. As a result, tourists sometimes get lost even with navigation. The HPA can predict the next place tourists will go and help them get to their potential destinations. Comparing the results of the predictions of individual spatial choices with crowded areas and potential crowded areas, congestion warning information could be sent to tourists who may prefer to those areas rather than to all tourists. Thus, the quality of tourist behavior and the tourist experience can eventually be optimized.

\subsection{Attraction/destination planning and management}

The heuristic prediction algorithm (HPA) can also provide support for conventional attraction and destination planning and management. In this paper, only 111 GPS tracking path data for the Summer Palace were used to show how to use the HPA method operationally. 
The HPA has many more potential applications when dealing with big data, and the prediction results of big data could be applied directly in attraction and destination planning and management. The overall quantity, location, and time arrangement of facilities and services could be predicted according to tourists' movement data. Combined with demographic information, the results of tourist flow predictions can be further analyzed according to gender, age, or other attributes, which may affect tourism product design and facility supply. Of the 111 tourists in the Summer Palace case study, 100 visited Spot 13 and 71 visited Spot 22, but few went to Spot 1, Spot 2, or Spot 8. In term of the duration time, tourists stayed more than half an hour at Spot 13 (60.4 minutes), Spot 3 (45.3 minutes), Spot 4 (44.8 minutes), Spot 22 (39.4 minutes), and Spot 24 (31.6 minutes). However, their visits were relatively short in other areas of the Summer Palace. The scale of tourists is related to the scale of facilities and services, and the duration time of tourists is related to the forms of facilities and services. According to the prediction results of the distribution of tourist flow at the Summer Palace, the supply of facilities and services in different areas could be correspondingly adjusted. Furthermore, popular tourist routes at the Summer Place could be identified. In the Summer Palace case study, 45 of the 111 tourists chose the combination of "Spot 13 + Spot 22," while 37 of these 45 tourists (20 males, 11 females, and 6 of unknown gender) chose to leave the Summer Palace after visiting these two areas rather than continue their visit. This kind of combination should draw the attention of attraction managers, as new tourism route design products could be explored according to tourists' behavior preferences.

\subsection{Real-time crowd control}

Tourist flow is composed of individual tourists. Based on the prediction results for individual tourists' spatial choices, the HPA can also be applied to forecast the potential time, space, and degree of congestion that may occur in the future; this would be valuable information for real-time crowd control. Crowd crushes may occur at high-level attractions, especially during holidays and at large-scale festival events, and can cause hundreds of fatalities. As shown in Fig. 12, the prediction results for the tourist distribution at the Summer Palace at 11:00, 11:30, 12:00, and 12:30 can be calculated using the HPA based on the historical data of tourists' spatial-temporal behavior. Potential crowd areas can be identified at regular intervals using real-time big data. In addition, the degree of congestion can be assessed according to the number of tourists within a certain scale area, and various appropriate countermeasures can be taken. For example, early warning information can be released on LCD screens and via multimedia tools to guide the flow to safe areas. If crowd rushes reach a certain degree of danger, compulsory measures are necessary to ensure tourist 
security.

\section{Conclusions and future research}

In this paper, we focused on a prediction method to identify tourists' next place of tourist attraction, which can benefit decision making and lead to proactivity rather than reactivity. Our research developed a heuristic prediction algorithm to predict the potential place of individual tourists that can result in a better trade-off between prediction accuracy and efficiency. GPS data collected from 111 tourists at the Summer Palace in Beijing, China were used to validate the method. The experimental results suggest that our algorithm has significantly higher prediction power and accuracy than existing methods. Furthermore, the HPA can be expanded from location prediction for individual tourists to predicting tourist distributions.

Our results significantly contribute to the current literature while also providing a series of guidelines to practitioners in management. First, our study offers a new perspective to improve the current algorithm. Although research has typically focused on developing effective algorithms to predict tourist behaviour, and thus help managers, neglect of the shortcomings in the $\mathrm{N}$-dimensional Markov model has tended to lower prediction power (Chen, et al., 2011; Landry, et al., 2013; Xia, et al., 2009; Xia, et al., 2011). Compared with prior studies, our results typically improve both the power and accuracy of prediction. Second, predicting intra-attraction tourist movements and distributions has a significant role in personalized LBS, tourist attraction administration, and real-time crowd control. This study expands the application of tourist movements in three ways in the context of big data, where movement data can be collected more easily: (1) This method can enhance management capability, that is, precision marketing, potential crowed prevention, etc.; (2) the proposed method offers policymakers a powerful tool to optimize resource allocation and service facility layout; and (3) the method can improve tourists' experience by providing them with real-time tour recommendations based on their behavioral preferences and the experiences of previous tourists.

In the future, to improve prediction accuracy, factors that affect tourists' movement choices should be considered, and tourist characteristics such as gender, age, and travel models, should be included when mining the candidate trajectories. In addition, further exploration of the applications of the HPA can be undertaken; these include the combination of the prediction of arrival times and durations at different attractions. 


\section{References}

Ashbrook, D., \& Starner, T. (2003). Using GPS to learn significant locations and predict movement across multiple users. Personal and Ubiquitous Computing, 7, 275-286.

Batty, M. (1997). Predicting where we walk. nature, 388, 19-20.

Bauder, M. (2014). Using GPS Supported Speed Analysis to Determine Spatial Visitor Behaviour. International Journal of Tourism Research.

Ben-Akiva, M. E., \& Lerman, S. R. (1985). Discrete choice analysis: theory and application to travel demand (Vol. 9): MIT press.

Campbell, C. (1967). An approach to research in recreational geography. Occasional Papers, 85-90.

Chen, L., Lv, M., \& Chen, G. (2010). A system for destination and future route prediction based on trajectory mining. Pervasive and Mobile Computing, 6, 657-676.

Chen, L., Lv, M., Ye, Q., Chen, G., \& Woodward, J. (2011). A personal route prediction system based on trajectory data mining. Information Sciences, 181, 1264-1284.

Coles, T., \& Hall, M. (2006). Editorial: The geography of tourism is dead. Long live geographies of tourism and mobility. Current Issues in Tourism, 9, 289-292.

Connell, J., \& Page, S. J. (2008). Exploring the spatial patterns of car-based tourist travel in Loch Lomond and Trossachs National Park, Scotland. Tourism Management, 29, 561-580.

Cresswell, T. (2006). On the move: mobility in the modern western world: Taylor \& Francis.

De Cantis, S., Ferrante, M., Kahani, A., \& Shoval, N. (2016). Cruise passengers' behavior at the destination: Investigation using GPS technology. Tourism Management, 52, 133-150.

Debbage, K. G. (1991). Spatial behavior in a Bahamian resort. Annals of Tourism Research, 18, 251-268.

Dong, M., \& He, D. (2007). Hidden semi-Markov model-based methodology for multi-sensor equipment health diagnosis and prognosis. European Journal of Operational Research, $178,858-878$.

Editorial Committee of Beijing Chorography. (2004). Beijing Chorography, World Cultural Heritage Volume, Summer Palace. Beijing: Beijing Press.

Fennell, D. A. (1996). A tourist space-time budget in the Shetland Islands. Annals of Tourism Research, 23, 811-829.

Gonzalez, M. C., Hidalgo, C. A., \& Barabási, A.-L. (2008). Understanding individual human mobility patterns. nature, 453, 779-782.

Hallo, J. C., Beeco, J. A., Goetcheus, C., McGee, J., McGehee, N. G., \& Norman, W. C. (2012). GPS as a method for assessing spatial and temporal use distributions of nature-based tourists. Journal of Travel Research, 51, 591-606.

Hannam, K., Butler, G., \& Paris, C. M. (2014). Developments and key issues in tourism mobilities. Annals of Tourism Research, 44, 171-185.

Hannam, K., Sheller, M., \& Urry, J. (2006). Editorial: Mobilities, immobilities and moorings. Mobilities, 1, 1-22. 
Huang, X., \& Wu, B. (2012). Intra-attraction tourist spatial-temporal behaviour patterns. Tourism Geographies, 14, 625-645.

Hwang, Y.-H., Gretzel, U., \& Fesenmaier, D. R. (2006). Multicity trip patterns: Tourists to the United States. Annals of Tourism Research, 33, 1057-1078.

Kenett, D. Y., \& Portugali, J. (2012). Population movement under extreme events. Proceedings of the National Academy of Sciences, 109, 11472-11473.

Laasonen, K. (2005). Clustering and prediction of mobile user routes from cellular data. In Knowledge Discovery in Databases: PKDD 2005 (pp. 569-576): Springer.

Landry, S. J., Chen, X. W., \& Nof, S. Y. (2013). A decision support methodology for dynamic taxiway and runway conflict prevention. Decision Support Systems, 55, 165-174.

Lau, G., \& McKercher, B. (2006). Understanding tourist movement patterns in a destination: A GIS approach. Tourism and Hospitality Research, 7, 39-49.

Lew, A., \& McKercher, B. (2006). Modeling tourist movements: A local destination analysis. Annals of Tourism Research, 33, 403-423.

Li, X., Meng, F., \& Uysal, M. (2008). Spatial pattern of tourist flows among the Asia-Pacific countries: An examination over a decade. Asia Pacific Journal of Tourism Research, 13, 229-243.

Lin, L.-Z., \& Yeh, H.-R. (2013). Analysis of tour values to develop enablers using an interpretive hierarchy-based model in Taiwan. Tourism Management, 34, 133-144.

Lu, X., Bengtsson, L., \& Holme, P. (2012). Predictability of population displacement after the 2010 Haiti earthquake. Proceedings of the National Academy of Sciences, 109, 11576-11581.

Lu, X., Wetter, E., Bharti, N., Tatem, A. J., \& Bengtsson, L. (2013). Approaching the limit of predictability in human mobility. Scientific reports, 3, 1-9.

Lue, C.-C., Crompton, J. L., \& Fesenmaier, D. R. (1993). Conceptualization of multi-destination pleasure trips. Annals of Tourism Research, 20, 289-301.

Mckercher, B., \& Lau, G. (2008). Movement patterns of tourists within a destination. Tourism Geographies, 10, 355-374.

McKercher, B., Shoval, N., Ng, E., \& Birenboim, A. (2012). First and repeat visitor behaviour: GPS tracking and GIS analysis in Hong Kong. Tourism Geographies, 14, 147-161.

McKercher, B., Shoval, N., Park, E., \& Kahani, A. (2015). The [Limited] Impact of Weather on Tourist Behavior in an Urban Destination. Journal of Travel Research, 54, 442-455.

O'Connor, A., Zerger, A., \& Itami, B. (2005). Geo-temporal tracking and analysis of tourist movement. Mathematics and Computers in Simulation, 69, 135-150.

Oppermann, M. (1995). A model of travel itineraries. Journal of Travel Research, 33, 57-61.

Orellana, D., Bregt, A. K., Ligtenberg, A., \& Wachowicz, M. (2012). Exploring visitor movement patterns in natural recreational areas. Tourism Management, 33, 672-682.

Pettersson, R., \& Zillinger, M. (2011). Time and space in event behaviour: tracking visitors by GPS. Tourism Geographies, 13, 1-20.

Shoval, N., \& Isaacson, M. (2007). Sequence alignment as a method for human activity analysis in space and time. Annals of the Association of American geographers, 97, 
282-297.

Shoval, N., McKercher, B., Ng, E., \& Birenboim, A. (2011). Hotel location and tourist activity in cities. Annals of Tourism Research, 38, 1594-1612.

Smallwood, C. B., Beckley, L. E., \& Moore, S. A. (2012). An analysis of visitor movement patterns using travel networks in a large marine park, north-western Australia. Tourism Management, 33, 517-528.

Song, C., Qu, Z., Blumm, N., \& Barabási, A.-L. (2010). Limits of predictability in human mobility. science, 327, 1018-1021.

Song, L., Kotz, D., Jain, R., \& He, X. (2006). Evaluating next-cell predictors with extensive Wi-Fi mobility data. IEEE Transactions on Mobile Computing, 5, 1633-1649.

Tideswell, C., \& Faulkner, B. (1999). Multidestination travel patterns of international visitors to Queensland. Journal of Travel Research, 37, 364-374.

Tsai, C.-Y., \& Chung, S.-H. (2012). A personalized route recommendation service for theme parks using RFID information and tourist behavior. Decision Support Systems, 52, 514-527.

Vu, T. H. N., Ryu, K. H., \& Park, N. (2009). A method for predicting future location of mobile user for location-based services system. Computers \& industrial engineering, 57, 91-105.

Wall, G. (1978). Competition and complementarity: A study in park visitation. International Journal of Environmental Studies, 13, 35-41.

Xia, J. C., \& Arrowsmith, C. (2005). Managing scale issues in spatio-temporal movement of tourists modelling. In International Congress on Modelling and Simulation (pp. 162-169).

Xia, J. C., Evans, F. H., Spilsbury, K., Ciesielski, V., Arrowsmith, C., \& Wright, G. (2010). Market segments based on the dominant movement patterns of tourists. Tourism Management, 31, 464-469.

Xia, J. C., Zeephongsekul, P., \& Arrowsmith, C. (2009). Modelling spatio-temporal movement of tourists using finite Markov chains. Mathematics and Computers in Simulation, 79, 1544-1553.

Xia, J. C., Zeephongsekul, P., \& Packer, D. (2011). Spatial and temporal modelling of tourist movements using Semi-Markov processes. Tourism Management, 32, 844-851.

Yang, Y., Fik, T., \& Zhang, J. (2013). Modeling sequential tourist flows: where is the next destination? Annals of Tourism Research, 43, 297-320.

Yavaş, G., Katsaros, D., Ulusoy, Ö., \& Manolopoulos, Y. (2005). A data mining approach for location prediction in mobile environments. Data \& Knowledge Engineering, 54, 121-146. 